\title{
Maggioranti e minoranti delle soluzioni delle equazioni paraboliche.
}

Memoria di Bruno Pini (a Cagliari).

Sunto. - Si definiscono e si caratierizzano le funzioni che, relativamente alle soluzioni dell'equazione $\partial^{2} u / \partial x^{2}+\partial u / \partial y=0$, sono le analoghe delle funzioni superarmoniche e subar. moniche; di esse si stabiliscono alcune proprietà dalle quali si trae poi partito per caratterizzare le soluzioni dell' equazione anzidetta. Lo stesso è successivamente fatto in relazione all equarione $\partial^{4} u / \partial x^{4}+2 \partial^{3} u / \partial x^{2} \partial y+\partial^{2} u / \partial y^{2}=0$.

Com'è noto, una funzione $u(P)$, ad esempio continua in un campo $A$ dello spazio euclideo ad $n$ dimensioni, si dice super-armonica (sub-armonica; se, comunque si fissi un dominio $D$ contenuto in $A$ per il quale esista la soluzione del problema ordinario di DIRICHLET $\Delta v=0$ in $D-\mathscr{F} D$ e $v=u$ su $\mathscr{F} D$, riesce $u \geq v(u \leq v)$ in ogni punto di $D$. Tali funzioni sono caratterizzate dal segno del $\Delta u$, quando di questo si può parlare, e, in generale, dal segno del

$$
\max _{r \rightarrow 0} \lim _{r \rightarrow 0}\left[\mu_{0}(u, P, r)-u(P)\right] / r^{2} \quad\left(\min _{r \rightarrow 0} \lim \left[\mu_{0}(u, P, r)-u(P)\right] / r^{2}\right),
$$

intendendosi con $\mu_{0}(u, P, r)$ la media dei valori di $u$ sulla ipersuperficie sferica di centro $P$ e raggio $r\left({ }^{1}\right)$. I risultati conseguiti per le funzioni super-armoniche (sub-armoniche) sono stati parzialmente estesi alle funzioni super-armoniche (sub-armoniche) d'ordine $p\left({ }^{2}\right)$, cioè alle funzioni $u(P)$ tali che, per ogni dominio $D$ per cui esiste la soluzione del problema di RIQUIEK $\Delta^{p} v=0$ in $D-\mathscr{F} D$ e $\Delta^{i} v=\Delta^{i} u$ su $\mathfrak{F} D$ per $i=0,1, \ldots, p-1$, riesce $u \geq v(u \leq v)$ in ogni punto di $D$.

Una trattazione del tipo di quella svolta per le funzioni super-armoniche (sub-armoniche) può presumibilmente farsi in relazione alle funzioni appartenenti a una certa classe le quali siano caratterizzate dal soddisfare una certa proprietà di media del tipo

$$
u(P)=\int_{s(P)} K(P, Q) u(Q) d Q
$$

(1) Cfr. p. es. T. RADo, Subharmonic functions, "Ergebnisse der Mathematik "; Berlin 1925 e New York 1949.

(2) M. NICOLAsco, Les fonctions polyharmoniques, Actualités scientifiques et indu. strielles *, n. 331, Paris 1936. 
ove $s(P)$ sia una ipersuperficie chinsa contenente $P$ nel suo interno, o alla quale $P$ eventualmente appartenga, e $K(P, Q)$ una funzione mai negativa tale che

$$
1=\int_{(P)} K(P, Q) d Q
$$

Nella presente Nota consideriamo le soluzioni dell' equazione

$$
\mathscr{T R}(u)=\partial^{2} u / \partial x^{2}+\partial u / \partial y=0
$$

e le funzioni che per esse sono le analoghe delle super-armoniche (sub-armoniche), estendendo a quelle alcune proprietà di queste. Servendoci poi di tali proprieta caratterizziamo le soluzioni di (1) mediante certi operatori che estendono al caso in esame l'operatore di BLAschke $\left({ }^{3}\right)$ per le funzioni armoniche.

I risultati conseguiti in relazione all' equazione (1) vengono poi estesi in relazione alle soluzioni dell' equazione iterata

$$
\mathscr{N K}^{2}(u)=\mathscr{N K}(\mathscr{T K}(u))=\partial^{4} u / \partial x^{4}+2 \partial^{3} u / \partial x^{2} \partial y+\partial^{2} u / \partial y^{2}
$$

L'estensione al caso di più di due variabili e di operatori iterati d'ordine superiore al secondo non deve presentare alcuna difficoltà.

\section{I. - Maggioranti e minoranti delle soluzioni di $\mathscr{K}(u)=0$.}

1. In tutto il seguito con la locuzione dominio normale intenderemo un dominio del tipo $\chi_{1}(y) \leq x \leq \chi_{2}(y), a \leq y \leq b$ con $\chi_{1}(y)<\chi_{2}(y)$ per $a \leq y \leq b$, essendo $\chi_{1}(y)$ e $\chi_{2}(y)$ due funzioni continue con le derivate prime. Se $D$ è un siffatto dominio $\theta(x, y)$ un suo punto, con $D_{y}$ intenderemo il prodotto di $D$ per il semipiano $\eta \geq y$ e con $S_{y}$ il prodotto di $\mathscr{F} D$ per $\eta \geq y$ se $y>a$, mentre con $S$ intenderemo la $\mathfrak{F} D$ privata della porzione di caratteristica $y=a, \chi_{1}(a)<x<\chi_{2}(a)$.

Diremo poi che una funzione $u(P)$ è della classe $C^{(n)}$ in $A$ se è ivi continua insieme alle sue derivate dei primi $n$ ordini; diremo invece che dे della classe $K^{(n)}$ se è continua insieme alle derivate che figurano in $\mathfrak{K}$, $\mathfrak{O K}^{2}, \ldots, \mathfrak{N C}^{n}$.

Indichiamo con

$$
U(Q, P)=\left\{\begin{array}{lll}
(\eta-y)^{-1 / 2} \exp \left[-(\xi-x)^{2} / 4(\eta-y)\right] & \text { per } \eta>y \\
0 & \text { per } \eta \leq y
\end{array}\right.
$$

$(P \equiv(x, y), Q \equiv(\xi, \eta))$ la soluzione fondamentale dell'equazione del calore.

(3) W. BLASCHKE, Ein Mittelwertsatz und eine Kennzeichnende Eigenschaft des logarithmischen Potentials, "Leipziger Berichte ", 68 (1916). 
Fissato. $P$, e un numero positivo $r$, indichiamo poi con $\mathcal{C}(P, r)$ la curva

$$
\left\{\begin{array}{l}
\xi=x+\sqrt{2} r \operatorname{sen} \theta \sqrt{\lg \left(1 / \operatorname{sen}^{2} \theta\right)} \quad-\pi / 2 \leq \theta \leq \pi / 2 \\
\eta=y+r^{2} \operatorname{sen}^{2} \theta
\end{array}\right.
$$

che è una linea di livello della $(3)$, e con $\mathfrak{D}(P, r)$ il dominio che ha $\mathcal{C}(P, r)$ per completa frontiera.

Sia $D$ un dominio normale. Fissato un punto $T(\sigma, \tau)$ di $D-S$, sia $V(Q, T)$ la soluzione di $\mathscr{L}(V)=\partial^{2} V / \partial \xi^{2}-\partial V / \partial \eta=0$, che coincide con $U(Q, T)$ sulle porzioni laterali e inferiore della $\mathscr{F} D_{\tau}$; indichiamo poi con $G_{i}(Q, T)$ la funzione di GREen relativa al dominio $D$ :

$$
G_{1}=-\frac{1}{2 \sqrt{\pi}}(U-V) \text {. }
$$

Allora, se $u(P)$ è della classe $K^{(t)}$ in un campo $A$ e $D$ è un dominio normale appartenente ad $A$, si ha

$$
u(T)=\int_{S_{\tau}}\left(u \frac{\partial G_{1}}{\partial \xi} d \eta+u G_{1} d \xi\right)+\iint_{D_{\tau}} G_{1} \mathscr{N}(u) d Q
$$

(intendendo che $S_{\tau}$ sia percorso positivamente). Nelle stesse ipotesi sulla $u(P)$, fissato un punto $P$ di $A$ e un numero positivo $r$ tale che $\mathscr{D}(P, r) \subset A$, dalla (6), prendendo $D \equiv \mathscr{D}_{\tau}(P, r)-\mathscr{D}_{y+r_{-\varepsilon}}(P, r)$, per $T \rightarrow P$ dall' interno di $\mathscr{D}(P, r)$ ed $\varepsilon \rightarrow 0$, si ha

$$
\begin{aligned}
& u(P)=\frac{1}{\sqrt{2 \pi}} \int_{-\pi / 2}^{\pi / 2}(u)_{\mathcal{C}(P, r)} \cos \theta \sqrt{\lg \left(1 / \operatorname{sen}^{2} \theta\right)} d \theta- \\
& -\sqrt{\frac{2}{\pi}} \int_{0-\pi / 2}^{r} \int_{\pi / 2}^{\pi / 2}\left(\frac{1}{\rho}-\frac{1}{r}\right) \frac{\rho^{2} \operatorname{sen}^{2} \theta \cos \theta}{\sqrt{\lg \left(1 / \operatorname{sen}^{2} \theta\right)}} \mathfrak{T C}(u) d \rho d \theta,
\end{aligned}
$$

notando che $V \rightarrow 1 / r$ poichò $U(Q, T)=1 / r$ su $\mathcal{C}(P, r)\left({ }^{4}\right)$. Posto

$$
\mu_{11}(u, P, r)=\frac{1}{\sqrt{2 \pi}} \int_{-\pi / 2}^{\pi / 2}(u)_{\mathcal{C}(P, r)} \cos \theta \sqrt{\lg \left(1 / \mathrm{sen}^{2} \theta\right)} d \theta
$$

( $\left.{ }^{4}\right)$ Prendendo ad esempio $Z^{H}=(x, \tau)$, la $V(Q, T)$ si mantiene limitata per $T \rightarrow P$ mentre

$\left|\frac{\partial U}{\partial \xi} d \eta+U d \xi\right|_{\mathcal{Q}(P, \gamma)}=\sqrt{2}\left(\frac{\tau-y}{2 z} \lg \frac{r^{2}}{z+\tau-y}+\frac{1}{2}\right) \exp \left(-\frac{\tau-y}{2 z} \lg \frac{r^{2}}{z+\tau-y}\right) \frac{|d z|}{\sqrt{z \lg \frac{r^{2}}{z+\tau-y}}}$
avendo posto $z=r^{2} \operatorname{sen}^{2} \theta-(\tau-y)$, onde

$$
\left|\frac{\partial U}{\partial \xi} d \eta+U d \xi\right|_{\mathfrak{C}_{(P, r)}} \sim \frac{|d z|}{\sqrt{\boldsymbol{z}|\lg z|}}
$$


si ha che:

a) Una soluzione regolare $d i \mathfrak{K}(u)=0$ in un campo $A$, verifica la

$$
u(P)=\mu_{n}(u, P, r)
$$

in ogni punto $P$ di $A$ e per ogni $r$ ammissibile (tale cioè che $\mathfrak{D}(P, r) \subset A$ ).

Reciprocamente: una funzione $u(P)$ continua in $A$ è ivi soluzione regolare di $\mathfrak{T R}(u)=0$ se per ogni punto $P$ di $A$ e per ogni $r$ ammissibile vale la (9).

La dimostrazione $\partial$ pressoche immediata se si suppone che $u$ sia in $A$ della classe $K^{(1)}\left({ }^{5}\right)$.

Nella sola ipotesi della continuità di $u$, la proposizione enunciata riesce una conseguenza di quanto sarà provato al $n$. 3 .

A caratterizzare le soluzioni di $\mathscr{T}(u)=0$, anzichè usare la formola di media periferica (9), si puo usare la formola di media superficiale

$$
u(P)=\mu_{1}(u, P, r)
$$

con

$$
\mu_{1}(u, P, r)=\frac{1}{r^{2}} \sqrt{\frac{2}{\pi}} \int_{0}^{r} \int_{-\pi / 2}^{\pi / 2} u \rho \cos \theta \sqrt{\lg \left(1 / \operatorname{sen}^{2} \theta\right)} d \rho d \theta=\frac{2}{r^{2}} \int_{0}^{r} \rho \mu_{0}(u, P, \rho) d \rho .
$$

Si ha che:

a') Una soluzione regolare di $\mathscr{T C}(u)=0$ in un campo A, verifica la $\left(9^{\prime}\right)$ in ogni punto $P$ di $A$ e per ogni $r$ ammissibile.

Reciprocamente: una funzione $u(P)$ continua in $A$ è ivi soluzione di $\operatorname{TK}(u)=0$ se per ogni punto $P$ di $A$ e per ogni valore ammissibile di $r$ sussisle la $\left(9^{\prime}\right)$.

2. Assegnata nel campo $A$ una funzione continua $v(P)$ e fissato in $A$ un dominio normale $D$, diremo associata della $v$ rispetto a $D$ la funzione $u$ tale che $\mathscr{T}(u)=0$ in $D-S$ e $u=v$ su $S$. Ciò posto, chiameremo $\mathscr{K}$-prevalente (ЮK-subvalente) in $A$ una funzione $v(P)$ continua in $A$ tale che, comunque si scelga in $A$ un dominio normale $D$, detta $u(P)$ la funzione associata della $v$ rispetto a $D$, riesce $v \geq u(v \leq u)$, in tutto $D$.

Si ha che:

b) Condizione necessaria e sufficiente affinchè una funzione $v$ della

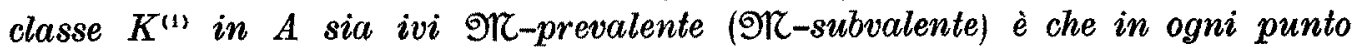
di A sia

$$
\mathscr{K}(v) \leq 0
$$

$$
(\mathfrak{T R}(v) \geq 0) \text {. }
$$

(5) B. PINI. Sulle equazioni a derivate parziali lineari del secondo ordine in due variabili, di tipo parabolico, "Annali di Mat. pura e appl. ", (4), XXXII (19à1). 
B. PINI : Maggioranti e minoranti delle soluzioni delle equazioni, ecc.

Consideriamo un dominio normale $D$ interno ad $A$ e sia $u$ la funzione associata. Dalla (6) si ha

$$
v(P)-u(P)=\iint_{D_{y}} G_{i}(Q, P) \mathscr{K}(v(Q)) d Q,
$$

onde l'affermazione è conseguenza del fatto che $G_{1} \leq 0$. Per provare ciò basta osservare ohe in $D_{y+\varepsilon}(0<\varepsilon<b-y), G_{1}$ è soluzione regolare di $\left\{\left(G_{1}\right)=0\right.$ onde il suo massimo $e$ il suo minimo sono raggiunti in punti della porzioni laterali $\theta$ inferiore di $\mathcal{F} D_{y+\varepsilon}$.

Sulle prime è $G_{1}=0$; sulla seconda è $U>0$ mentre $0<V<m(\varepsilon)$ con $m(\varepsilon) \rightarrow 0$ per $\varepsilon \rightarrow 0$; pertanto il $\max G_{1}$ sa $D_{y}$ \& zero.

Segue che:

c) Condizione necessaria e sufficiente affinchè una funzione $v(P)$ della classe $\mathbb{K}^{(1)}$ in $A$ sia ivi $\mathfrak{T}$-prevalente (פK-subvalente) è che, per ogni punto $P$ di $A$ e per tutti $i$ valori ammissibili di $r$, sia

$$
v(P) \geq \mu_{0}(v, P, r) \quad\left(v(P) \leq \mu_{0}(v, P, r)\right) .
$$

Questa proposizione segue immediatamente dalla b) e da (7).

Analogamente, sostituendo la media superficiale a quella periferica:

$\left.\mathrm{c}^{\prime}\right)$ Condizione necessaria e sufficiente affinchè $v(P)$ della classe $K^{(1)}$ in $A$ sia ivi Or -aprevalente (ЮT-subvalente) è che sia

$$
v(P) \geq \mu_{1}(v, P, r) \quad\left(v(P) \leq \mu_{1}(v, P, r)\right)
$$

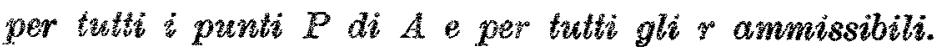

Questo dwe ultimg proposimioni sussistono anche nella sola ipotesi di continaita della $v\left(P\right.$ ) come risulta dalle seguenti d) $\left.\Theta d^{\prime}\right)$ :

d) Condizione necessaria e sufficiente affinchè la funzione $v(P)$ continua in A sia ivi 9 K-prevalente (פK-subvalente) $\grave{e}$ che per ogni punto $P$ di A la media $\mu_{0}(v, P, r)$ sia funzione non crescente (non decrescente) di $r$.

Supposta della classe $K^{(1)}$, dalla (7) si ha

$$
\frac{d}{d r} \mu_{0}(v, P, r)=\frac{1}{r^{2}} \sqrt{\frac{2}{\pi}} \int_{0}^{r} \int_{\pi / 2}^{\pi / 2} \rho^{2} \frac{\operatorname{sen}^{2} \theta \cos \theta}{\sqrt{\lg \left(1 / \operatorname{sen}^{2} \theta\right)}} \mathscr{M}(v) d \rho d \theta
$$

di qui segue la necessità della condizione in base alla b).

Per la sufficienza basta osservare che dall' essere $\mu_{0}(v, P, \rho) \geq \mu_{0}(v, P, r)$ $\left(\mu_{0}(v, P, \rho) \leq \mu_{0}(v, P, r)\right)$ per $r>p$, segue al limite per $\rho \rightarrow 0, v(P) \geq \mu_{0}(v P, r)$ $\left(v(P) \leq \mu_{0}(v, P, r)\right)$ con che si ricade nella $\left.c\right)$. 
Mostriamo ora la necessita della condizione nell'ipotesi che $v$, supposta soltanto continua, sia per esempio $\mathfrak{K}$-prevalente. Sia $r_{1}>r_{2}>0$; indichiamo con $T$ un punto interno a $\mathscr{D}\left(P, r_{2}\right)$, per esempio $T=(x, \tau)$. Consideriamo $\mathrm{i}$ domini $\mathscr{D}_{\tau}\left(P, r_{1}\right)-\mathscr{D}_{y+r_{2}^{2}-\varepsilon}\left(P, r_{1}\right) \in \mathfrak{D}_{\tau}\left(P, r_{2}\right)-\mathscr{D}_{y+r_{2}^{2}-\varepsilon}\left(P, r_{2}\right)$, con $\varepsilon$ sufficientemente piccolo, e indichiamo brevemente con $S_{1 \varepsilon}$ ed $S_{2 \varepsilon}$ le corrispondenti porzioni $S$ di frontiera. Detta $u(P)$ la funzione associata della $v$ rispetto al dominio $\mathscr{D}_{\tau}\left(P, r_{1}\right)-\mathscr{D}_{y+r_{1}^{2}-\varepsilon}\left(P, r_{1}\right)$, si ha per la $(6)$

$$
\int_{S_{1 \varepsilon}}\left(v \frac{\partial G_{1}^{\prime}}{\partial \xi} d \eta+v G_{1}^{\prime} d \xi\right)=\int_{S_{q \varepsilon}}\left(u \frac{\partial G_{1}^{\prime \prime}}{\partial \xi} d r_{1}+u G_{1}^{\prime \prime} d \xi\right)
$$

indicando con $G_{1}^{\prime}$ e $G_{1}^{\prime \prime}$ le funzioni di GreEs corrispondenti ai due domini.

Facciamo ora tendere $T$ a $P$ dall' interno di $\mathscr{D}\left(P, r_{2}\right)$. Detti $A_{1} B_{1}$ e $A_{2} B_{2}$ i tratti rettilinei (percorsi positivamente) di $S_{1 \varepsilon}$ ed $S_{z \varepsilon}$, si ha

$$
\begin{gathered}
\left.\frac{1}{\sqrt{2 \pi}} \int_{\widetilde{A_{3} P B_{1}}} v \cos \theta \sqrt{\lg \left(1 / \operatorname{sen}^{2} \theta\right)} d \theta-\int_{\overline{A_{1} B_{1}}} v G_{1}^{\prime} d \xi=\frac{1}{\sqrt{2 \pi}} \int_{\overline{A_{2} P B_{2}}} u \cos \theta \sqrt{\lg \left(1 / \operatorname{sen}^{2} \theta\right.}\right) d \theta- \\
\left.-\int_{\overline{A_{2} B_{2}}} u G_{1}^{\prime \prime} d \xi \leq \frac{1}{\sqrt{2 \pi}} \int_{\overline{A_{2} P B_{2}}} v \cos \theta \sqrt{\lg \left(1 / \operatorname{sen}^{2} \theta\right.}\right) d \theta-\int_{\overline{A_{2} B_{2}}} v G_{1}^{\prime \prime} d \xi
\end{gathered}
$$

tenendo presente che $v \geq u$ e che $G_{1} \leq 0$. Di qui, per $\varepsilon \rightarrow 0$, si ha infine

$$
\mu_{0}\left(v, P, r_{1}\right) \leq \mu_{0}\left(v, P, r_{2}\right)
$$

La sufficienza, nel caso caso che $v(P)$ si supponga soltanto continua, segue dal successivo n. 3 .

$\left.d^{\prime}\right)$ Condizione necessaria e sufficiente affinchè $v(P)$ continua in $A$ sia ivi $\mathfrak{K}$-prevalente (OK-subvalente) $\dot{e}$ che la media superficiale $\mu_{1}(v, P, r)$ in ogni punto $P$ di A sia funzione non crescente (non decrescente) di $r$.

La sufficienza si prova come in d) se $v$ è della classe $K^{(1)}$; se $v$ è solo continua, essa segue dal successivo n. 3. La necessità, per esempio nel caso che $v$ sia $\mathfrak{T K}$-prevalente, segue da

$$
\begin{gathered}
\frac{d}{d r} \mu_{1}(v, P, r)=2\left[\frac{\mu_{0}(v, P, r)}{r}-\frac{2}{r^{3}} \int_{0}^{r} \rho \mu_{0}(v, P, \rho) d \rho\right] \leq \\
\leq \frac{2}{r}\left[\mu_{0}(v, P, r)-\frac{2}{r^{2}} \int_{0}^{r} \rho \mu_{0}(v, P, r) d \rho\right]=0 .
\end{gathered}
$$


3 Se $v(P)$ è una funzione della classe $K^{(1)}$ in $A$, in ogni punto $P$ di $A$ si ha

$$
\begin{aligned}
& \lim _{r \rightarrow 0} \frac{3 \sqrt{3}\left[\mu_{0}(v, P, r)-v(P)\right]}{r^{2}}=\operatorname{Tr}(v(P)) \\
& \lim _{r \rightarrow 0} \frac{6 \sqrt{3}\left[\mu_{1}(v, P, r)-v(P)\right]}{r^{2}}=\operatorname{TK}(v(\dot{P})) .
\end{aligned}
$$

Pertanto se sulla $v$ si fa ad esempio la sola ipotesi della continuità, gli operatori indicati nei primi membri di $(13)$ e $\left(13^{\prime}\right)$ riescono delle generalizzazioni dell' operatore $\mathfrak{i}$, cosi come gli operatori di Blaschere e di Privaloff costituiscono delle generalizzazioni del laplaciano.

Poniamo

$$
\begin{aligned}
& \max _{r \rightarrow 0} \lim _{r \rightarrow \overline{3}} \frac{\left.3 \sqrt{\mu_{1}}(v, P, r)-v(P)\right]}{r^{2}}=\operatorname{OK}_{\mathrm{n}}{ }^{\prime \prime}(v, P) \\
& \min _{r \rightarrow 0} \frac{3 \sqrt{3}\left[\mu_{i 1}(v, P, r)-v(P)\right]}{r^{2}}=\operatorname{TK}_{0}{ }^{\prime}(v, P) .
\end{aligned}
$$

Si ha:

e) Condizione necessaria e sufficiente affinchè la funzione $v(P)$, continua

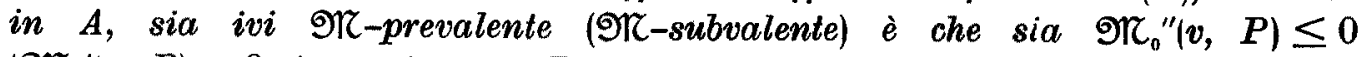
$\left(\mathfrak{O K}_{\text {. }}{ }^{\prime}(v, P) \geq 0\right.$ ) in ogni punto $P$ di $A$.

La necessità segue dai risultati del n. 2. Proviamo la sufficienza, per esempio nel primo caso (nel secondo si ragiona in modo analogo). Supponiamo dapprima che sia sempre $\mathfrak{K K}_{0}{ }^{\prime}(v, P)<0$. Allora $v$ è $\mathfrak{T K}$-prevalente. Se ciò non fosse, esisterebbe un dominio normale $D$ in $A$ tale che, detta $u$ la fun. zione associata della $v$ rispetto a $D$, in $D-S$ vi sarebbero dei punti ove $v<u$; sia $P_{0}$ un punto ove $v-u$ raggiunge il suo minimo; sarà allora $v\left(P_{0}\right)-u\left(P_{0}\right) \leq \mu_{0}\left(v-u, P_{0}, r\right)$ per tutti gli $r$ abbastanza piccoli e quindi

$$
0 \leq \mathfrak{T K}_{0}^{\prime \prime}\left(v-u, P_{0}\right)=\mathfrak{T K}_{0}^{\prime \prime}\left(v, P_{0}\right)-\mathscr{T K}_{0}{ }^{\prime \prime}\left(u, P_{0}\right)=\mathfrak{T K}_{0}{ }^{\prime \prime}\left(v, P_{0}\right)
$$

il che contraddice l'ipotesi. Supposto poi che sia $\operatorname{TK}_{0}^{\prime \prime}(v, P) \leq 0$ in ogni punto $P$ di $A$, nell'ipotesi che $A$ sia limitato, oppure riferendoci a un arbitrario dominio limitato contenuto in $A$ nel caso che questo non sia limitato, consideriamo la successione di funzioni

$$
v_{n}(P)=v(P)-x^{2} / 2 n \quad(n=1,2, \ldots)
$$


Allora, trovandoci nelle condizioni in eui ei si era posti prima, si può affermare che le $v_{n}$ sono tutte $\mathscr{T}$-prevalenti. Fissato ad arbitrio in $A$ un dominio normale $D$, detta $u_{n}(P)$ la funzione associata della $v_{n}(P)$ rispetto a $D$, poichè la successione delle funzioni $v_{n}(P)$ converge uniformemente a $v(P)$, la successione $u_{n}(P)$ convergerà alla funzione $u$ associata della, $v$ rispetto a $D$; pertanto dall'essere $v_{n} \geq u$, per ogni $n$, segue $v \geq u$.

In modo del tutto simile, posto

$$
\begin{aligned}
& \max _{r \rightarrow 0} \frac{6 \sqrt{3}\left[\mu_{1}(v, P, r)-v(P)\right]}{r^{2}}=\mathfrak{N K}_{1}^{\prime \prime}(v, P) \\
& \min _{r \rightarrow 0} \frac{6 \sqrt{3}\left[\mu_{1}(v, P, r)-v(P)\right]}{r^{2}}=\mathfrak{I K}_{1}^{\prime}(v, P),
\end{aligned}
$$

si ha che:

e') Condizione necessaria e sufficiente affinchè la funzione v(P), continua in $A$, sia ivi $\mathscr{T}$-prevalente $(\mathscr{N K}$-subvalente $)$ è che sia $\mathscr{\mathfrak { K }} "(v, P) \leq 0$ $\left(\mathscr{K}_{1}{ }^{\prime}(v, P) \geq 0\right)$ in ogni punto $P$ di $A$.

Infine da e) e da e') si deduce che:

f) Condizione necessaria e sufficiente affinchè la funzione v(P), continua in $A$, sia ivi soluzione (regolare) $d i \mathfrak{T K}(v)=0$, è che in ogni punto $P$ di A sia

$$
\lim _{r \rightarrow 0} \frac{\mu_{0}(v, P, r)-v(P)}{r}=0 \quad\left(\lim _{r \rightarrow 0} \frac{\mu_{1}(v, P, r)-v(P)}{r^{2}}=0\right)\left(^{6}\right) .
$$

\section{II. - Maggioranti e minoranti delle soluzioni di $\mathfrak{T K}^{2}(u)=0$.}

4. Se $u(P)$ e $v(P)$ sono due funzioni della classe $K^{(2)}$ in $A$ e $\Delta$ è un dominio appartenente ad $A$, sussiste la formola di reciprocità

$$
\begin{aligned}
& \iint_{\Delta}\left[v \mathscr{N} \mathcal{K}^{2}(u)-u \mathcal{L}^{2}(v)\right] d Q=
\end{aligned}
$$

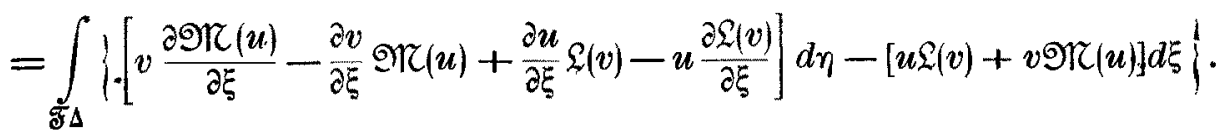

(6) Si noti che tutte le volte che esiste il primo dei limiti (15)), esiste anche il secondo di tali limiti ed è eguale a un mezzo del primo. Infatti, detto $\lambda$ tale limite, si ha $\mu_{\theta}(v, P, r)=v(P)+\lambda r^{2}+o\left(r^{2}\right)$, onde

$$
\frac{\mu_{1}(v, P, r)-v(P)}{r^{2}}=\frac{1}{r^{4}}\left[2 \int_{0}^{r} \mu_{0}(v, P, \rho) \rho d \rho-r^{2} v(P)\right]=\frac{\lambda}{2}+\frac{2}{r^{4}} \int_{0}^{r} \rho o\left(\rho^{2}\right) d \rho \underset{r \rightarrow 0}{\longrightarrow} \frac{\lambda}{2} .
$$


Fissiamo in $V$ un dominio normale $D$ e sia $T$ un punto di $D-S$. Poniamo

$$
G_{2}(Q, T)=\iint_{D_{n, \tau}} G_{1}(Q, M) G_{1}(M, T) d M
$$

essendo $G_{i}$ la funzione (5) e $D_{n, \tau}$ il dominio prodotto di $D$ per la strisoia $\tau \leq y \leq \eta$.

$G_{1}$ e $G_{2}$ si annullano quando il punto $Q$ si trova sulle porzioni laterali e inferiore della $\mathscr{F} D_{\eta, \tau}$. Per un noto risultato di GEVREY si ha poi $\mathscr{S}\left(G_{2}\right)=G_{1}$.

Se $u(P)$ è in $A$ della classe $K^{(2)}$, applicando la (16) al dominio $D_{\tau}-\mathscr{D}(T, \varepsilon)$ e facendo tendere $\varepsilon$ a zero, si ha

(18) $u(T)=\int_{S_{\tau}}\left\{\left[\frac{\partial G_{2}}{\partial \xi} \mathscr{T K}(u)+u \frac{\partial G_{1}}{\partial \xi}\right] d \eta+\left[u G_{1}+\mathscr{T K}(u) G_{2}\right] d \xi\right\}+\int_{D_{\tau}} \int_{z} G_{z} \mathscr{N}{ }^{\prime \prime}(u j d \xi d \eta$ (ove $S_{\tau}$ s'intende percorsa positivamente). Nelle stesse ipotesi sulla $u$, se $\mathfrak{T}(P, r)$ appartiene ad $A$, preso per $D$ il dominio $\mathscr{D}_{\tau}(P, r)-\mathscr{D}_{y+r_{-\varepsilon}}(P, r)$, per $T \rightarrow P$ dall' interno di $\mathfrak{D}(P, r)$ ed $\varepsilon \rightarrow 0$, osservando che $G_{2} \rightarrow \frac{1}{2 \sqrt{\pi}}[U(Q, P)-1 / r](\eta-y)$, si ottiene

$$
\begin{aligned}
& u(P)=\frac{1}{\sqrt{2 \pi}} \int_{-\pi / 2}^{\pi / 2}(u)_{\mathfrak{e}(P, r)} \cos \theta \sqrt{\lg \left(1 / \operatorname{sen}^{2} \theta\right)} d \theta- \\
& -\frac{r^{2}}{\sqrt{2 \pi}} \int_{-\pi / 2}^{\pi / 2}(\mathscr{T K}(u))_{\mathcal{C}(P, r)} \operatorname{sen}^{2} \theta \cos \theta \sqrt{\lg \left(1 / \operatorname{sen}^{2} \theta\right)} d \theta+ \\
& +\sqrt{\frac{2}{\pi}} \int_{0}^{r / 2} \int_{-\pi / 2}^{\pi / 2}\left(\frac{1}{\rho}-\frac{1}{r}\right) \frac{\rho^{4} \operatorname{sen}^{4} \theta \cos \theta}{\sqrt{\lg \left(1 / \operatorname{sen}^{2} \theta\right)}} \mathscr{K C}^{2}(u) d \rho d \theta .
\end{aligned}
$$

Posto

$$
\begin{gathered}
M_{0}(u, P, r)=\frac{1}{\sqrt{2 \pi}} \int_{-\pi / 2}^{\pi / 2}(u)_{\mathcal{C}(P, r)} \cos \theta \sqrt{\lg \left(1 / \operatorname{sen}^{2} \theta\right)} d \theta- \\
-\frac{r^{2}}{\sqrt{2 \pi}} \int_{-\pi / 2}^{\pi / 2}(\mathscr{T H}(u))_{\mathcal{C}(P, r)} \operatorname{sen}^{2} \theta \cos \theta \sqrt{\lg \left(1 / \operatorname{sen}^{2} \theta\right)} d \theta
\end{gathered}
$$

si verifica immediatamente che:

a) Condizione necessaria e sufficiente affinchè una funzione u(P) della classe $K^{(2)}$ in $A$ sia ivi soluzione di $\mathscr{K}^{2}(u)=0$, è che per ogni punto $P$ di $A$ e per ogni valore (ammissibile) di $r$ sia

$$
u(P)=M_{0}(u, P, r) \text {. }
$$


Anzichè caratterizzare le soluzioni di $\mathfrak{T K}^{2}(u)=0$ mediante la formola di media (21), ̀̀ possibile farlo con una formola di media ove figuri solo la $u$; però questa sarà di tipo superficiale e non più periferico come la precedente.

Dalla formola di reciprocità

$$
\iint_{\Delta}[v \mathscr{R}(u)-u \mathcal{L}(v)] d Q=\int\left[\left(v \frac{\partial u}{\partial \xi}-u \frac{\partial v}{\partial \xi}\right) d \eta-u v d \xi\right]
$$

prendendo $\mathscr{D}(P, r)$ al posto di $\Delta$, si ottiene

$$
\begin{gathered}
\int_{\mathfrak{D}(P, r)}[v \mathscr{N}(u)-u \mathcal{L}(v)] d Q=\sqrt{2} r^{2} \int_{-\pi / 2}^{\pi / 2}\left(v \frac{\partial u}{\partial \rho}-u \frac{\partial v}{\partial \rho}\right)_{\mathcal{C}(P, r)} \cos \theta \sqrt{\lg \left(1 / \operatorname{sen}^{2} \theta\right)} d \theta+ \\
+2 \sqrt{2} r \int_{-\pi 2}^{\pi / 2}\left(u \frac{\partial v}{\partial \theta}\right)_{\mathcal{C}(P, r)} \operatorname{sen} \theta \sqrt{\lg \left(1 / \operatorname{sen}^{2} \theta\right)} d \theta
\end{gathered}
$$

Questa sussiste anche se, essendo $u$ della classe $K^{(1)}$, si pone $v=\lg \left(1 / \operatorname{sen}^{2} \theta\right)$. Si ottiene

$$
\begin{aligned}
& \int_{0-\pi / 2}^{r} \int_{0}^{\pi / 2} \mathscr{T K}(u) \rho^{2} \operatorname{sen}^{2} \theta \cos \theta \sqrt{\lg \left(1 / \operatorname{sen}^{2} \theta\right)} d \rho d \theta= \\
& =\int_{0-\pi / 2}^{r} \int_{/ 2}^{\pi / 2} u\left[1+\lg \left(1 / \operatorname{sen}^{2} \theta\right)\right] \frac{\cos \theta}{\sqrt{\lg \left(1 / \operatorname{sen}^{2} \theta\right)}} d \rho d \theta+
\end{aligned}
$$

$+\frac{r^{2}}{2} \int_{-\pi / 2}^{\pi / 2}\left(\frac{\partial u}{\partial \rho}\right)_{\mathcal{C}(P, r)} \cos \theta\left[\lg \left(1 / \operatorname{sen}^{2} \theta\right)\right]^{3 / 2} d \theta-2 r \int_{-\pi / 2}^{\pi / 2}(u)_{\mathcal{C}_{(P, r)}} \cos \theta \sqrt{\lg \left(1 / \operatorname{sen}^{2} \theta\right)} d \theta$.

Integrando la (21) da zero ad $r$, tenendo presente la formola ora stabilita, si ha

$$
\begin{gathered}
r u(P)=-\frac{1}{\sqrt{2 \pi}} \int_{0-\pi / 2}^{r} \int_{-2 / 2}^{\pi / 2} \frac{\cos \theta}{\sqrt{\lg \left(1 / \operatorname{sen}^{2} \theta\right)}} d \rho d \theta-\frac{r^{2}}{2 \sqrt{2 \pi}} \int_{-\pi / 2}^{\pi / 2}\left(\frac{\partial u}{\partial \rho}\right)_{\mathcal{C}(P, r)} \cos \theta\left[\lg \left(1 / \operatorname{sen}^{2} \theta\right)\right]^{3 / 2} d \theta+ \\
\left.+\frac{2 r}{\sqrt{2 \pi}} \int_{-\pi / 2}^{\pi / 2}(u)_{\mathcal{C}(P, r)} \cos \theta \sqrt{\lg \left(1 / \operatorname{sen}^{2} \theta\right.}\right) d \theta .
\end{gathered}
$$

Integrando nuovamente da zero ad $r$ ed eseguendo opportune integrazioni per parti e poi nuovamente integrando da zero ad $r$, si ottiene in 
definitiva

$$
\begin{gathered}
u(P)=\frac{3}{r^{3} \sqrt{2 \pi}} \int_{0-\pi / 2}^{r \pi / 2} u\left\{2 \rho(r-\rho)\left[1+\lg \left(1 / \operatorname{sen}^{2} \theta\right)\right]^{2}-\right. \\
\left.-\rho^{2} \lg ^{2}\left(1 / \operatorname{sen}^{2} \theta\right)-r^{2}+\rho^{2}\right\} \frac{\cos \theta}{\sqrt{\lg \left(1 / \operatorname{sen}^{2} \theta\right)}} d \rho d \theta .
\end{gathered}
$$

Indicando con $M_{1}(u, P, r)$ il secondo membro, si ha la formola di media

$$
u(P)=M_{i}(u, P, r)
$$

la quale caratterizza, al pari della $(21)$, le soluzioni di $\mathfrak{K R}^{\mathfrak{a}}(u)=0$. Precisamente :

$\left.a^{\prime}\right)$ Condizione necessaria e sufficiente affinchè una funzione $u(P)$, della classe $K^{(2)}$ in $A$, sia ivi soluzione di $\mathscr{T K}^{2}(u)=0$, è che in ogni punto $P$ di $A$ e per tutti gli $r$ ammissibili valga la (21').

Dai risultati del successivo n. 6 seguirà poi che:

$\left.a^{\prime \prime}\right)$ Se $u(P)$ è in $A$ una funzione della classe $K^{(1)}$ e se in ogni punto $P d i A$ e per tutti gli $r$ ammissibili sussiste la (21) oppure la $\left(21^{\prime}\right)$, allora è $\mathfrak{T}^{2}(u)=0$.

5. Se $v(P)$ è una funzione della classe $K^{(1)}$ in $A$, fissato un dominio normale $D$ in $A$, chiameremo associata della $v(P)$ rispetto a $D$ la funzione $u(P)$ tale che $\mathfrak{K C}^{2}(u)=0$ in $D-S, u=v$ e $\mathfrak{T}(u)=\mathscr{K}(v)$ su $S$.

Diremo poi che una funzione $v(P)$ della classe $K^{(1)}$ in $A$ è ivi $\mathfrak{K}^{2}-$ prevalente (ЮK(2-subvalente) se, fissato ad arbitrio un dominio normale $D$ in $A$ e detta $u(P)$ la funzione associata della $v(P)$, riesce $v(P) \geq u(P)(v(P) \leq u(P))$ in ogni punto $P$ di $D$.

Si ha che:

b) Condizione necessaria e sufficiente affinchè la funzione $v(P)$, di classe $K^{(2)}$ in $A$, sia ivï $\mathfrak{K}^{2}$-prevalente $\left(\mathscr{T K}^{2}-\right.$ subvalente) è che per ogni punto $P$ di $A$ sia

$$
\mathfrak{T K}^{2}(v) \geq 0 \quad\left(\operatorname{TK}^{2}(v) \leq 0\right) .
$$

Se $D$ è un dominio normale contenuto in $A$ e $u(P)$ la funzione ivi associata della $v(P)$, dalla $(18)$ segue

$$
v(P)-u(P)=\iint_{D_{y}} G_{2}\left(Q, P \mid \mathscr{V K}^{2}(v) d Q\right.
$$

e quindi l'asserto segue immediatamente dall'osservazione che, in base alla (17), essendo $G_{1} \leq 0$, ̀े $G_{2} \geq 0$.

Segue che:

c) Condizione necessaria e sufficiente affinchè una funzione $v(P)$, della

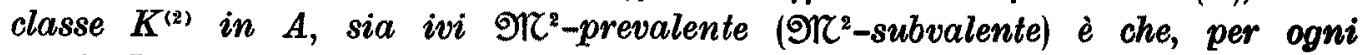
punto $P$ di $A$ e per tutti $i$ valori ammissibili $d i r$, sia

$$
v(P) \geq M_{0}(v, P, r) \quad\left(v(P) \leq M_{0}(v, P, r)\right) .
$$


Cio segue dalla formola (19) e dalla precedente proposizione b), Analogamente :

$\left.\mathrm{c}^{\prime}\right)$ Condizione necessaria e sufficiente affinchè una funzione $v(P)$, della classe $K^{(2)}$ in $A$, sia ivi $\mathfrak{K}^{2}$-prevalente $\left(\mathfrak{K}^{2}-\right.$ subvalente) $\dot{e}$ che per ogni punto $P$ di $A$ e per tutti $i$ valori ammissibili di $r$, sia

$$
v(P) \geq M_{1}(v, P, r) \quad\left(v(P) \leq M_{1}(v, P, r)\right) .
$$

Le proposizioni c) e $e^{\prime}$ ) sono assorbite dalle successive e) ed $e^{\prime}$ ).

Premettiamo che:

d) Se $v(P)$ è in $A$ della classe $K^{(1)}$ e verifica la prima o la seconda delle (23'), allora $\mathfrak{K}(v)$ è $\mathfrak{K}$-subvalente oppure $\mathfrak{K}$-prevalente.

Se $f(P)$ è una funzione definita in $A$, poniamo

$$
\omega^{(1)}(f, P, r)=\frac{1}{\pi r^{2}} \iint_{\xi^{2}+\eta^{2}<r^{2}} f(x+\xi, y+\eta) d \xi d \eta
$$

ogniqualvolta il cerchio di centro $P$ e raggio $r$ appartiene ad $A$ ed $f(P)$ dे ivi integrabile. Posto

$$
\omega^{(n)}(f, P, r)=\omega\left(\omega^{(n-1)}(f, Q, r), P, r\right)
$$

(essendo $Q$ un punto variabile sul cerchio di centro $P$ e raggio $r$ ) è noto che se $f$ ̀े della classe $C^{(m)}, \omega^{(1)}(f, P, r)$ è della classe $C^{(m+1)}$.

Ora se $v(P)$ verifica per esempio la prima delle $\left(23^{\prime}\right)$, si ha per ogni punto $P$ di $A$ e per ogni $r$ ammissibile

$$
v(x, y) \geq \frac{3}{r^{3} \sqrt{2 \pi}} \int_{0-\pi / 2}^{r} \int_{\pi / 2}^{\pi / 2} K(r, \rho, \theta) v\left(x+\sqrt{2} \rho \operatorname{sen} \theta \sqrt{\lg \left(1 / \operatorname{sen}^{2} \theta\right)}, y+\rho^{2} \operatorname{sen}^{2} \theta\right) d \rho d \theta
$$

ove il significato di $K(r, \rho, \theta)$ si deduce dalla (22). Perciò

$$
\omega^{(1)}\left(v, P, r_{1}\right) \geq \frac{3}{r^{3} \sqrt{2 \pi}} \iint_{\xi+\eta^{2}<r_{1}^{2}} \frac{1}{\pi r_{1}{ }^{2}}\left(\int _ { 0 - \pi / 2 } ^ { r } \int _ { y + \eta } ^ { \pi / 2 } K ( r , \rho , \theta ) v \left(x+\xi+\sqrt{2} \rho \operatorname{sen} \theta \sqrt{\lg \left(1 / \operatorname{sen}^{2} \theta\right.}\right.\right.
$$

ossia

$$
\begin{gathered}
\omega^{(1)}\left(v, P, r_{1}\right) \geq \frac{3}{r^{3} \sqrt{2 \pi}} \int_{0-\pi / 2}^{r} \int_{\left.y+\rho^{2} \operatorname{sen}^{2} \theta, r_{1}\right) d \rho d \theta .}^{\pi / 2} K(r, \rho, \theta) \omega^{(1)}\left(v, x+\sqrt{2} \rho \operatorname{sen} \theta \sqrt{\lg \left(1 / \operatorname{sen}^{2} \theta\right)},\right.
\end{gathered}
$$

Il procedimento si può iterare. Pertanto in ogni dominio contenuto in $A$, prendendo $r_{1}$ abbastanza piccolo, si riconosce che $\omega^{(2)}\left(v, P, r_{1}\right)$ əे una funzione della classe $K^{(2)}$ e, verificando la prima delle $\left(23^{\prime}\right)$, ¿̀ $\mathfrak{K}^{2}$-prevalente. Ne segue che $\mathscr{T C}\left(\omega^{(2)}\left(v, P, r_{1}\right)\right)$ è $\mathscr{K}$-subvalente. Ma, essendo $v$ della classe $K^{(1)}$, 
le successioni

$$
\omega^{(2)}(v, P, 1 / n), \quad \mathscr{N K}\left(\omega^{(3)}(v, P, 1 / n)\right)=\omega^{(2)}(\mathscr{T K}(v), P, 1 / n), \quad n>\bar{n}
$$

convergono uniformemente rispettivamente a $v$ e $\mathscr{T}(v)$ in ogni dominio limitato appartenente ad $A\left({ }^{7}\right)$. Pertanto dall' essere

$$
\mathscr{T K}\left(\omega^{(2)}(v, P, 1 / n)\right) \leq \mu_{0}\left(\mathscr{T K}\left(\omega^{(2)}(v, Q, 1 / n)\right), P, r\right)
$$

(dove $Q$ è un punto variabile su $\mathfrak{C}(P, r)$ ) segue

$$
\mathscr{N R}(v(P)) \leq \mu_{n}(\mathscr{N K}(v), P, r)
$$

e questa, in base ai risultati di 1 , assicura l" asserto.

Cio posto, proviamo che:

e) Condizione necessaria e sufficiente affinchè la funzione $v(P)$, di classe $K^{(1)}$ in $A$, sia ivi $\mathfrak{N}^{2}-p r e v a l e n t e\left(\mathfrak{N}^{2}-\right.$ subvalente), è che la media $M_{0}(v, P, r)$ in ogni punto $P$ di $A$ sia funzione non crescente (non decrescente) di $r$.

Supposto dapprima che $v(P)$ sia di classe $K^{(2)}$, la sufficienza si prova come in $3, \mathrm{~d}$ ).

La necessità segue dall' osservare che

$$
\frac{d}{d r} M_{0}(v, P, r)=-\frac{1}{r^{2}} \sqrt{\frac{2}{\pi}} \int_{0}^{r} \int_{\pi / 2}^{\pi / 2} \frac{\rho^{4} \operatorname{sen}^{4} \theta \cos \theta}{\sqrt{\lg \left(1 / \operatorname{sen}^{2} \theta\right)}} \operatorname{NC}^{2}(v) d \rho d \theta
$$

Nel caso che $v(P)$ si supponga solo di classe $K^{(1)}$, la sufficienza segue dal successivo n. 6. Per quanto riguarda la necessita, supposto $v(P)$ per esempio $\mathfrak{K}^{2}$-prevalente, ragionando per assurdo, esisterebbero un punto ${ }^{\prime}$ e due numeri $r_{1}$ ed $r_{2}, r_{1}>r_{2}>0$, tali che, essendo $\mathscr{D}\left(P, r_{1}\right)$ contenuto in $A$, sarebbe

$$
M_{0}\left(v, P, r_{1}\right)>M_{0}\left(v, P, r_{2}\right)
$$

$\mathrm{D}^{\prime}$ altra parte, essendo $\omega^{(2)}(v, P, 1 / n) \mathscr{N K}^{\prime}$-prevalente (per $\left.n>\bar{n}\right)$, sarebbe

$$
M_{0}\left(\omega^{(2)}(v, Q, 1 / n), P, r_{1}\right) \leq M_{0}\left(\omega^{(2)}(v, Q, 1 / n), P, r_{2}\right)
$$

ciò che, per la convergenza uniforme di $\omega^{(2)}(v, P, 1 / n)$ a $v$ per $n \rightarrow \infty$, contraddice la precedente.

$\left.\mathrm{e}^{\prime}\right)$ Condizione necessaria e sufficiente affinchè $v\left(P^{2}\right)$, di classe $K^{(1)}$ in $A$, sia ivi $\mathfrak{O K}^{2}$-prevalente $\left(\mathfrak{N R}^{2}-\right.$ subvalente $)$ è che la media $M(v, P, r)$ in ogni punto $P$ di $A$ sia funzione non crescente (non decrescente) di $r$.

(T) Fissato un dominio limitato $D$ interno ad $A$, prendendo $r_{1}$ abbastanza piccolo ed $\bar{n}$ abbastanza grande, è evidente che al variare di $P$ in $D$ e di $Q$ nel cerchio di centro $P$ e raggio $\langle 1 / \bar{n}, Q$ varierà in un dominio limitato interno ad $A$. 
Poichè

$$
M_{1}(v, P, r)=\frac{6}{r^{3}} \int_{0}^{r} d r_{1} \int_{0}^{r_{1}} d r_{2} \int_{0}^{r_{2}} M_{0}(v, P, \rho) d \rho
$$

si ha

$$
\frac{d}{d r} M_{1}(v, P, r)=-\frac{3}{r^{4}} \int_{0}^{r}\left(3 \rho^{2}-4 r \rho+r^{2}\right) M_{0}(v, P, \rho) d \rho,
$$

come si riconosce effettuando successive integrazioni per parti. Ora, posto

$$
f(r)=\int_{0}^{r}\left(3 \rho^{2}-4 r \rho+r^{2}\right) M_{0}(v, P, \rho) d \rho
$$

si ha

$$
f^{\prime}(r)=2 \int_{0}^{r}(r-2 \rho) M_{0}(v, P, \rho) d \rho, \quad f^{\prime \prime}(r)=2 \int_{0}^{r} M_{0}(v, P, \rho) d \rho-2 r M_{0}(v, P, r) .
$$

Poichè per e) è $M_{0}(v, P, r) \leq M(v, P, \rho)$ per $r>\rho$, segue $f^{\prime \prime}(r) \geq 0$ onde $f^{\prime}(r)$ è funzione non decrescente e poichè $f^{\prime}(0)=0$, \&े $f^{\prime}(r) \geq 0$; ne segue che $f(r)$ ̀े funzione non decrescente e poichè $f(0)=0$, è $f(r) \geq 0$. Pertanto $\frac{d}{d r} M(v, P, r) \leq 0$. Ciò prova la necessità. La sufficienza segue dal successivo n. 6.

6. Se $v(P)$ è in $A$ una funzione della classe $K^{(2)}$, in ogni punto $P$ di $A$ si ha

$$
\begin{aligned}
& \lim _{r \rightarrow 0} \frac{10 \vee \overline{5}\left[v(P)-M_{0}(v, P, r)\right]}{r^{4}}=\mathscr{N}^{2}(v(P) \\
& \lim _{r \rightarrow 0} \frac{350 \mathrm{~V} \overline{5}\left[v(P)-M_{1}(v, P, r)\right]}{r^{4}}=\mathfrak{N}^{2}(v(P))
\end{aligned}
$$

Perciò, qualora non esista l' $\mathfrak{T}^{2}(v)$ ordinario ma esistano i limiti che figurano al primo membro in $(24)$ e $\left(24^{\prime}\right)\left({ }^{8}\right)$, questi si potranno ritenere delle generalizzazioni dell' $\mathfrak{T C}^{2}(v)$. Si noti che mentre l'operatore al primo membro di (24) si può considerare per funzioni $v(P)$ della classe $K^{(1)}$, l'operatore a

(8) Si noti che se esiste il limite indicato dalla (24), esiste ed è uguale ad esso, anche il limite indicato dalla : $\left.24^{\prime}\right)$. Infatti, detto $\lambda$ tale limite, si ha $M_{0}=v-\frac{r^{4}}{10 \sqrt{5}} \lambda+o\left(r^{4}\right)$, onde

$$
\frac{350 \sqrt{5}\left[v(P)-M_{1}(v, P, r)\right]}{r^{4}}=\frac{350 \sqrt{5}}{r^{7}}\left[r^{3} v(P)-6 \int_{0}^{r} d r_{1} \int_{0}^{r_{1}} d r_{2} \int_{0}^{r_{2}}\left[v(P)-\frac{r^{4} \lambda}{10 \sqrt{5}}+o\left(r^{4}\right)\right] d p\right] \underset{r \rightarrow 0}{\rightarrow} \lambda .
$$


primo membro di (24) si può considerare anche per funzioni soltanto continue. Però, mentre la condizione

$$
\lim _{r \rightarrow 0} \frac{v(P)-M_{1}(v, P, r)}{r^{4}}=0
$$

¿̀ evidentemente necessaria perchè sia $\mathfrak{K}^{2}(v)=0$, essa non è sufficiente. Per esempio, sia $A$ il campo $-1<x<1,-1<y<1$, e sia

$$
v(P)=\left\{\begin{array}{lll}
x^{2}+y & \text { per } y \geq 0 \\
x^{2}-y & \text { per } y<0
\end{array}\right.
$$

Questa funzione non è in $A$ soluzione regolare di $\mathscr{T K}^{2}(v)=0$; ciò nonostante la (25) è soddisfatta in ogni punto $P$ di $A$. Lo stesso esempio prova come neppure la continuità di $v$ e di $\partial v / \partial x$, unitamente alla (25), sia suffi. ciente ad assicurare che $v(P)$ è soluzione regolare di $\mathfrak{W}^{2}(v)=0$.

Poniamo

Si ha:

$$
\begin{aligned}
& \max _{r \rightarrow 0} \frac{10 \sqrt{5}\left[v(P)-M_{0}(v, P, r)\right]}{r^{4}}=\mathfrak{N R}_{0}^{\prime \prime 2}(v, P) \\
& \min _{r \rightarrow 0} \frac{10 \sqrt{5}\left[v(P)-M_{0}(v, P, r)\right]}{r^{4}}=\mathfrak{N K}_{0}^{\prime 2}(v, P) .
\end{aligned}
$$

f) Condizione necessaria e sufficiente affinchè la funzione $v(P)$, di classe $K^{(1)}$ in $A$, sia iri $\mathfrak{O K}^{2}-$ prevalente $\left(\mathscr{O C}{ }^{2}-\right.$ subvalente), è che sia $\mathfrak{N K}_{0}^{\prime 2}(v, P) \geq 0$ $\left(\mathscr{O K}^{\prime \prime 2}(v, P) \leq 0\right)$ in ogni punto $P$ di $A$.

La necessità segue dal n. 5. Proviamo la sufficienza, per esempio per il primo caso (essendo il ragionamento in tutto simile per il secondo caso). Supponiamo dapprima che sia sempre $\mathfrak{N K}_{0}^{\prime 2}(v, P)>0$; allora $v$ \& $\mathfrak{N}^{2}$-prevalente. Se ciò non fosse, esisterebbe un dominio normale $D$ in $A$ tale che, essendo $u(P)$ la funzione associata della $v(P)$ rispetto a $D$, vi sarebbero dei punti di $D-S$ ove $v<u$; sia $P_{0}$ un punto ove $v-u$ ragginnge il suo minimo; sarà allora $\mathscr{N}(v)-\mathscr{O R}(u) \geq 0$ in $P_{0}$. Ora, per quanto si è provato in d), la $\mathscr{K}(v)$ è $\mathscr{K}$-subvalente e quindi $\mathscr{N}(v(P)) \leq \mu_{0}(\mathscr{N}(v), P, r)$ per tutti gli $r$ abbastanza piccoli; di qui la contraddizione se si suppone che valga sempre il segno <. Supposto poi che sia $\mathfrak{N K}_{0}^{\prime 2}(v, P) \geq 0$ e $\mathfrak{N K}(v(P)) \leq \mu_{0}(\mathscr{N R}(v), P, r)$, senza escludere che valga il segno di eguaglianza, consideriamo la successione di funzioni

$$
v_{n}(P)=v(P)+\frac{x^{4}}{4 ! n} \quad(n=1,2, \ldots),
$$

riferendoci a un arbitrario dominio limitato contenuto in $A$, qualora $A$ non sia limitato. Trovandoci ora nelle condizioni in cui ci si era posti prima, si può concludere che le $v_{n}(P)$ sono tutte $\mathscr{T}^{2}$-prevalenti. Ma le $v_{n}(P)$ e le $\mathscr{T C}\left(v_{n}\right)$ convergono uniformemente rispettivamente a $v(P)$ e a $\operatorname{GK}(v)$. Resta cosi provato che anche la $v(P)$ è $\mathfrak{K}^{2}$-prevalente. 
In modo del tutto simile, posto

$$
\begin{aligned}
& \max _{r \rightarrow 0} \frac{350 \sqrt{5}\left[v(P)-M_{1}(v, P, r)\right.}{r^{4}}=\mathfrak{N R}_{1}^{\prime 2}(v, P) \\
& \min _{r \rightarrow 0} \frac{350 \sqrt{5}\left[v(P)-M_{1}(v, P, r)\right]}{r^{4}}=\mathscr{N}_{1}^{\prime 2}(v, P)
\end{aligned}
$$

si ha che:

$\left.\mathrm{f}^{\prime}\right)$ Condizione necessaria e sufficiente affinchè la funzione $v(P), d i$

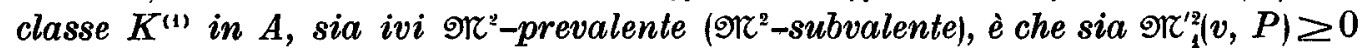
$\left(\mathfrak{T K}^{\prime \prime 2}(v, P) \leq 0\right)$ in ogni punto $P$ di $A$.

Infine da f) ed $f^{\prime}$ ) segue che:

g) Condizione necessaria e sufficiente affinchè la funzione $v(l)$, di classe $K^{(1)}$ in $A$, sia ivi soluzione (regolare) $d i \mathscr{N}^{2}(v)=0$, è che in ogni punto 1 di A sia

$$
\lim _{r \rightarrow 0} \frac{v(P)-M_{0}(v, P, r)}{r^{4}}=0 \quad\left(\lim _{r \rightarrow 0} \frac{v(P)-M_{4}(v, P, r)}{r^{4}}=0\right) .
$$

